\title{
PRÁTICAS DIGITAIS NAS UNIDADES DE I\&D EM PORTUGAL: UMA OBSERVAÇÃO PARCIAL DA ÁREA DAS HUMANIDADES
}

\author{
PRÁCTICAS DIGITALES EN LAS UNIDADES DE I+D EN \\ PORTUGAL: UNA OBSERVACIÓN PARCIAL DE LAS \\ HUMANIDADES
}

Jorge Revez *

\begin{abstract}
RESUMO:
Introdução: Em que patamar digital estão as Humanidades portuguesas? A resposta a esta pergunta exige uma aproximação ao quadro teórico das Humanidades Digitais e uma aplicação deste quadro à situação da investigação científica portuguesa. É o primeiro estudo cruzado (não restrito a uma disciplina) sobre as práticas digitais na área das Humanidades em Portugal. Objetivo: $O$ objetivo é analisar as práticas digitais na área das Humanidades em Portugal. Metodologia: Observámos uma amostra de 18 unidades de I\&D portuguesas que atuam na área das Humanidades e identificámos os seus projetos de investigação em curso. Consideraram-se os projetos mais relevantes para a construção de um inventário e categorização das evidências da incorporação de ferramentas digitais no trabalho científico e na criação dos seus produtos finais. Resultados: Os resultados da análise a 25 projetos ativos e a uma linha de investigação mostram que as práticas digitais estão disseminadas e comunicadas de forma incipiente. Conclusões: A transformação preconizada pelo campo das Humanidades Digitais ainda se encontra numa fase inicial em Portugal.
\end{abstract}

Palavras-chave: Humanidades Digitais. Investigação científica. Humanidades. Unidades de I\&D. Portugal.

Em que patamar digital estão as Humanidades portuguesas? Esta pergunta levanta um conjunto tão vasto de problemas que não podemos tentar enunciar uma resposta sem primeiro nos interrogarmos sobre a essência do que queremos perguntar. E mesmo a resposta terá de ser sempre provisória e * Doutorando em Ciência da Informação pela Universidade de Coimbra. E-mail: jrevez@campus.ul.pt 
aberta a contributos futuros. Hayles entrevistou alguns académicos acerca da sua relação com as tecnologias digitais e avançou uma preocupante estimativa: apenas $10 \%$ utilizavam estas ferramentas de uma forma construtiva e como uma mais-valia para a execução dos seus projetos de investigação (2012, p. 59). Esta estimativa aumentava com os jovens investigadores e professores, 0 que anunciava a transformação inevitável da academia em academia digital.

Neste sentido e olhando para o presente caso de estudo, questionarmos as práticas digitais das humanidades não é necessariamente o mesmo que tentar fazer uma avaliação da penetração teórico-prática das Humanidades Digitais (HD) na investigação que está a ser realizada em Portugal. Cremos que pode ser feita uma distinção entre o uso das chamadas novas tecnologias, com destaque para a computação e todas as ferramentas por si potenciadas, e a passagem para um outro nível, em que, por um lado, ocorre uma apropriação de um discurso e, por outro, sobrevém uma mudança no tipo de operações investigativas, com impacto óbvio na forma como interpretamos os fenómenos humanos e sociais.

Como afirmou Pereira, não se pode reduzir "a missão das Humanidades Digitais à mera aplicação de ferramentas e recursos digitais". Está em causa o debate e a experimentação de "novos modelos de compreensão da cultura e do mundo" (2015, p. 135). Zorich insistiu numa distinção semelhante, entre uso normal e transformativo, entre a utilização das tecnologias e os novos produtos e processos que transformam o conhecimento existente (2008, p. 4). Criticando o caráter dualista destas considerações, Mullen avançou que as HD não podem ser entendidas como a simples oposição às Humanidades tradicionais. O seu ethos é de inclusão e pode ser transformado em ferramenta de persuasão, integrando e promovendo aprendizagens mútuas (2010).

Num estudo recente sobre a relação das bibliotecas com as HD, Varner e Hswe descrevem a ruptura que se tem vindo a verificar nos últimos dez anos, encarando as HD como o resultado de um diálogo dinâmico entre as tecnologias emergentes e a investigação das humanidades (2016, p. 36-37). Neste diálogo, como Parry notou, as HD, enquanto transdisciplina de saberes e práticas, transportam a ideia de transformação. Não são apenas a interseção 
entre o substantivo humanidades e o adjetivo digitais. A questão é como o digital transformará a noção de humanidades (2012, p. 437). A omnipresença do fenómeno digital operou uma mudança na epistemologia e nas formas de trabalho académico. Não estamos no domínio exclusivo do uso pois é impossível distinguir o impacto das novas tecnologias - e a consequente omnipresença do digital - da forma como se faz hoje ciência.

Esta mudança não se limita assim à aplicação das tecnologias às Humanidades. Estamos perante um campo novo de possibilidades analíticas, uma lente crítica renovada e impossível de ser levada à prática antes da invenção da computação (PARRY, 2012, p. 431). As HD são uma área de saberes colaborativos, ancorada nas Humanidades, para as transformar em algo novo, multiplicando as formas epistemológicas de as conceber e analisar. Desta forma, as HD assumem uma componente transformadora em que a computação assume uma forma ontoteológica, criando uma nova constelação histórica de inteligibilidade (BERRY, 2012, p. 16).

Em 2011, num estudo internacional sobre a investigação nesta área (BULGER et al., 2011), os autores concluíam que o progresso era evidente quanto às práticas informacionais. Contudo eram deletadas barreiras como a falta de formação, suporte e normalização. Estes e outros obstáculos limitam a capacidade dos investigadores em estabelecer ligações e relações entre os diferentes recursos. Os resultados deste trabalho são claros quanto aos desafios que os académicos enfrentam, onde Portugal não é uma exceção. Alguns destes desafios são a necessidade de formação, incluindo a formação tecnológica para usar novas ferramentas e serviços, e de apoio continuado no seu uso; a necessidade de desenvolver e tornar sustentável (a longo prazo) a infraestrutura digital de apoio à investigação; e melhorar a capacidade de partilha de dados e interligação entre diferentes arquivos (BULGER et al., 2011, p. 7-8).

No caso de Portugal, Alves realizou um ponto da situação das HD, notando uma evolução lenta e marcada pela relação histórica que as humanidades têm estabelecido com as tecnologias digitais, mas com sinais claros que indiciam uma mudança positiva (2014). Anteriormente e usando 
uma metodologia próxima daquela que seguimos neste exercício, Guardado e Borges (2012) analisaram o caso das unidades de I\&D a atuar no campo da História, com resultados positivos quanto à adoção de práticas digitais, mesmo em comparação com a realidade internacional: "The major difference is related to patterns of collaboration. In a number of non-Portuguese projects, public participation is one of the main characteristics, while in Portugal the collaboration is mainly inside the scientific community, involving historians and other researchers" (p. 49-50).

Contactando diretamente as unidades de I\&D, Guardado e Borges também analisaram as revistas científicas portuguesas na área da história, com resultados positivos quanto ao início de uma mudança comportamental dos investigadores. As unidades de I\&D portuguesas, apesar da dimensão do país, têm valorizado o acesso livre eletrônico aos resultados da sua investigação, aproveitando a visibilidade oferecida pela Web para comunicar com um maior número de investigadores (2011, p. 240).

Um conjunto internacional de autores realizou um estudo sobre a experiência sócio acadêmica do Dia das Humanidades Digitais e observou as resistências do campo das Humanidades. Uma característica da emergência das HD em espanhol e em português é a tensão entre a resistência de assumir que as humanidades se definam pelo digital e $o$ interesse por agrupar-se e discutir sob essa terminologia (PRIANI SAISÓ et al., 2014, p. 16).

Querendo averiguar as evidências das práticas acantonadas nas HD mas também a assimilação do seu discurso, o que aqui se apresenta é um exercício e uma proposta de trabalho: explorar as práticas digitais nas unidades de I\&D portuguesas através dos projetos em curso. Esta proposta apresenta uma metodologia, parcelar e condicionada, mas que cremos suficiente para podermos traçar um retrato da adoção das HD em Portugal.

Quisemos assim realizar um exercício que nos aproximasse do discurso e das práticas adotadas nas unidades de I\&D em Portugal com o objetivo de os avaliar, e aí observarmos a presença de uma mudança em curso. E essa mudança é evidente, em maior ou menor grau, conforme os contextos de análise. 
Jorge Revez

Práticas digitais nas unidades de I\&D em Portugal: uma observação parcial da área das humanidades

\section{METODOLOGIA}

A avaliação das unidades de I\&D realizada entre 2013 e 2015 (pelo acordo celebrado entre a Fundação para a Ciência e Tecnologia [FCT] e a European Science Foundation ${ }^{1}$ ) foi o ponto de partida para a constituição da nossa amostra de estudo. Das 322 unidades avaliadas, foi atribuído um financiamento para 2015-2020 a 257, sendo 39 da área das Humanidades. Com estes dados, optámos por selecionar as 18 unidades melhor classificadas, às quais foi atribuída a nota de Excecional e Excelente, constituindo assim a nossa amostra (Quadro 1)

\begin{tabular}{|c|c|c|}
\hline Nome & Acrónimo & Fonte \\
\hline $\begin{array}{lr}\text { Center } & \text { for } \\
\text { General } & \text { and } \\
\text { Applied } & \\
\text { Linguistic } & \\
\text { Studies } & \end{array}$ & $\begin{array}{l}\text { CELGA- } \\
\text { ILTEC }\end{array}$ & http://celga.iltec.pt/projects.html \\
\hline $\begin{array}{lr}\text { Center } & \text { for } \\
\text { Psychology } & \text { at } \\
\text { University } & \text { of } \\
\text { Porto } & \end{array}$ & CPUP & http://www.fpce.up.pt/cpup/centro.html \\
\hline $\begin{array}{ll}\text { Centre } & \text { for } \\
\text { Comparative } & \\
\text { Studies } & \end{array}$ & CEC & http://www.comparatistas.edu.pt/en/research-groups/groups/ \\
\hline $\begin{array}{l}\text { Centre of } \\
\text { Portuguese } \\
\text { Literature }\end{array}$ & CLP & http://www.uc.pt/fluc/clp/inv/proj \\
\hline $\begin{array}{l}\text { Cognitive and } \\
\text { Behavioural } \\
\text { Centre for } \\
\text { Research and } \\
\text { Intervention }\end{array}$ & CINEICC & http://www.uc.pt/en/fpce/research/CINEICC/groups \\
\hline $\begin{array}{l}\text { Communication } \\
\text { and Society } \\
\text { Research Centre }\end{array}$ & CECS & http://www.comunicacao.uminho.pt/cecs/content.asp?startAt=2\&categorylD=613 \\
\hline $\begin{array}{l}\text { Ethnomusicology } \\
\text { Institute - Center } \\
\text { for Studies in } \\
\text { Music and Dance }\end{array}$ & INET-md & http://www.fcsh.unl.pt/inet/projectos/pagina.html \\
\hline $\begin{array}{l}\text { Glass } \\
\text { Ceramic for the } \\
\text { Arts }\end{array}$ & VICARTE & http://www.vicarte.org/research ccm.html \\
\hline $\begin{array}{l}\text { Institut for } \\
\text { Comparative } \\
\text { Literature }\end{array}$ & ILC & http://ilcml.com/projecto-estrategico/ \\
\hline $\begin{array}{l}\text { Institut of } \\
\text { Contemporary } \\
\text { History }\end{array}$ & $\mathrm{IHC}$ & http://www.ihc.fcsh.unl.pt/pt/investigacao/projectos-em-curso \\
\hline Interdisciplinary & ICArEHB & http://www.icarehb.com/index.php/projects \\
\hline
\end{tabular}

1

Cf. http://www.fct.pt/apoios/unidades/avaliacoes/2013/processo avaliacao.phtml.pt Inf. Inf., Londrina, v. 22, n. 3, p. $405-425$, set./out. 2017. http:www.uel.br/revistas/informacao/ 
Jorge Revez

Práticas digitais nas unidades de I\&D em Portugal: uma observação parcial da área das humanidades

\begin{tabular}{|l|l|l|}
\hline $\begin{array}{l}\text { Center for } \\
\text { Archaeology and } \\
\text { Evolution of } \\
\text { Human } \\
\text { Behaviour }\end{array}$ & & \\
\hline $\begin{array}{l}\text { Interdisciplinary } \\
\text { Center for } \\
\text { History, Cultures } \\
\text { and Societies }\end{array}$ & CIDEHUS & http://www.cidehus.uevora.pt/investigacao/projetos/projetos em curso \\
\hline $\begin{array}{l}\text { Interuniversity } \\
\text { Center for the } \\
\text { History of } \\
\text { Science and } \\
\text { Technology }\end{array}$ & CIUHCT & http://ciuhct.org/pt/sc/investigacao/projectos-liderados \\
\hline $\begin{array}{l}\text { Nova Institute of } \\
\text { Philosophy IFILNOVA }\end{array}$ & http://www.filnova.pt/pages/r-d-projects \\
\hline $\begin{array}{l}\text { Portuguese } \\
\text { Centre for Global } \\
\text { History CHAM }\end{array}$ & http://www.cham.fcsh.unl.pt/projectos.aspx \\
\hline $\begin{array}{l}\text { Research Center } \\
\text { in Psychology }\end{array}$ & CIPsi & http://www.psi.uminho.pt/Default.aspx?tabid=11\&pageid=53\&lang=pt-PT \\
\hline $\begin{array}{l}\text { Research Centre } \\
\text { for the Sociology } \\
\text { and Aesthetics of } \\
\text { Music }\end{array}$ & CESEM & $\underline{\text { http://cesem.fcsh.unl.pt/investigacao }}$ \\
\hline $\begin{array}{l}\text { William James } \\
\text { Center for } \\
\text { Research WJCR }\end{array}$ & http://wjcr.ispa.pt/pagina/research-areas-0 \\
\hline
\end{tabular}

Fonte: Elaborado pelo autor.

Através da observação, em Dezembro de 2015, da informação disponibilizada na Web pelas unidades, procurámos identificar os projetos ativos ou em curso e, na descrição de cada projeto, retirámos a informação que denotava a aplicação de práticas digitais. Para tal, foram criadas duas folhas de recolha de dados (Anexos A e B) para acolher os dados da observação nãoparticipante das páginas das unidades e dos projetos em curso. Seguimos assim a indicação fornecida por Guardado e Borges, após registarem algum insucesso no contato direto com estas organizações (2012, p. 47).

A deteção das evidências de elementos característicos do que tem sido acantonado no campo das HD não foi uma tarefa fácil pois não existe um inventário estabelecido de práticas ou aplicações. Estas são o resultado de múltiplas abordagens, de experiências inter e transdisciplinares, não se fechando num cânone ou num esquema delimitado. Por essa razão, várias tentativas de criação de uma "cartografia" das HD foram apresentadas por autores como Svensson, não sendo possível desenhar uma paisagem concreta mas apenas um modelo de relações ou compromissos (2010, pr. 173; 175).

De forma a organizarmos os dados e para lhes conferir uma estruturação semântica, optámos por atribuir etiquetas aos textos retirados das 
páginas das unidades e ainda os categorizámos por campos científicos (dentro da área das Humanidades e seguindo a lista utilizada pela FCT ${ }^{2}$ ). Naturalmente que este método, ao limitar-se à informação disponibilizada publicamente, não garante a análise exaustiva e detalhada dos objetivos, métodos, práticas, conteúdo e produtos finais dos referidos projetos. Apresentamos assim resultados que são apenas parciais e sempre condicionados pelas escolhas comunicacionais das unidades de I\&D.

Por outro lado, a estruturação não padronizada dos dados apresentados nos sítios Web não facilitou a recolha de informação, dado que a grelha de recolha de dados sofreu diversos ajustes no decurso da observação e nem sempre conseguiu integrar toda a informação que pode vir a ser importante para trabalhos futuros.

São limitações deste estudo: a análise parcial das unidades de I\&D financiadas (18 em 39 - 46\%); a desatualização dos sítios Web relativamente à planificação para 2015-2020; a profusão de informação sobre os projetos estar dispersa em sítios autónomos e por vezes ser indistinta entre projetos concluídos e ativos; a informação disponibilizada ser maioritariamente sumária, restrita por vezes a uma sinopse ou aos objetivos científicos, sem informação sobre atividades previstas, metodologias ou práticas digitais. Podíamos ter ainda contactado a FCT ou as próprias unidades de I\&D, de forma a obter mais informação, o que terá necessariamente de ser feito se a amostra for aumentada em estudos vindouros. Para um estudo qualitativo dos projetos era igualmente importante a obtenção de mais dados.

\section{ANÁLISE DOS DADOS}

Os dados recolhidos podem ser agrupados em quatro áreas distintas que a seguir se apresentam.

2 Cf. https://www.fct.pt/apoios/projectos/concursos/2012/docs/Dominios e Areas Cientificas C2012.pdf

Inf. Inf., Londrina, v. 22, n. 3, p. $405-425$, set./out. 2017. http:www.uel.br/revistas/informacao/ 
Práticas digitais nas unidades de I\&D em Portugal: uma observação parcial da área das humanidades

\subsection{Unidades, projetos ativos e práticas digitais}

Os 25 projetos e uma linha de investigação (que considerámos neste ponto como um projeto) analisados apresentam um retrato positivo - uma média de 1,3 projetos por cada unidade -, ainda que tímido, da implementação de práticas digitais. É possível constatar que existe uma distribuição desigual dessas evidências nas 18 unidades de I\&D (Quadro 2).

Quadro 2 - N. ${ }^{\circ}$ de projetos analisados em cada Unidade de I\&D

Fonte: Elaborado pelo autor.

\begin{tabular}{|c|c|}
\hline ACRÔNIMO & No PROJETOS ANALISADOS \\
\hline INET-md & 5 \\
\hline CHAM & 4 \\
\hline CLP & 3 \\
\hline CIUHCT & 3 \\
\hline CIDEHUS & 2 \\
\hline IFILNOVA & 2 \\
\hline CELGA-ILTEC & 1 \\
\hline CPUP & 1 \\
\hline CEC & 1 \\
\hline CECS & 1 \\
\hline ILC & 1 \\
\hline IHC & 1 \\
\hline CESEM & 1 \\
\hline CINEICC & 0 \\
\hline VICARTE & 0 \\
\hline ICArEHB & 0 \\
\hline CIPsi & 0 \\
\hline WJCR & 0 \\
\hline & \\
\hline
\end{tabular}

A categorização dos Projetos por área científica resultou num claro destaque de 3 áreas - História, Estudos Literários e Musicologia -, ainda que a diversidade seja igualmente importante para provar a disseminação das práticas digitais nas Humanidades portuguesas (Quadro 3). 
Práticas digitais nas unidades de I\&D em Portugal: uma observação parcial da área das humanidades

Quadro 3 - N. ${ }^{\circ}$ de projetos analisados por área científica (FCT)

\begin{tabular}{|c|c|}
\hline Área científica (FCT) & $\begin{array}{c}\text { N. }{ }^{\circ} \text { de } \\
\text { Projetos }\end{array}$ \\
\hline Arqueologia & 1 \\
\hline Artes Multimédia & 1 \\
\hline $\begin{array}{c}\text { Ciências da Comunicação e da } \\
\text { Informação }\end{array}$ & 1 \\
\hline $\begin{array}{c}\text { Estudos literários } \\
\text { Filosofia }\end{array}$ & 5 \\
\hline História & 2 \\
\hline História e Filosofia da Ciência e da \\
Tecnologia & 3 \\
\hline Linguística & 2 \\
\hline Música e Musicologia & 5 \\
\hline
\end{tabular}

Fonte: Elaborado pelo autor.

O confronto percentual dos projetos ativos e dos projetos analisados, i.e. aqueles que considerámos apresentarem evidências de práticas digitais ainda que nem todas se pudessem considerar como práticas transformativas demonstra o grau de penetração das HD que podemos considerar como inicial (Gráfico 1). Note-se que aqui considerámos apenas nove das 18 unidades de I\&D pois a outra metade não apresentava um inequívoco número de projetos ativos, como veremos adiante. 
Gráfico 1 - Comparação percentual dos projetos ativos/analisados

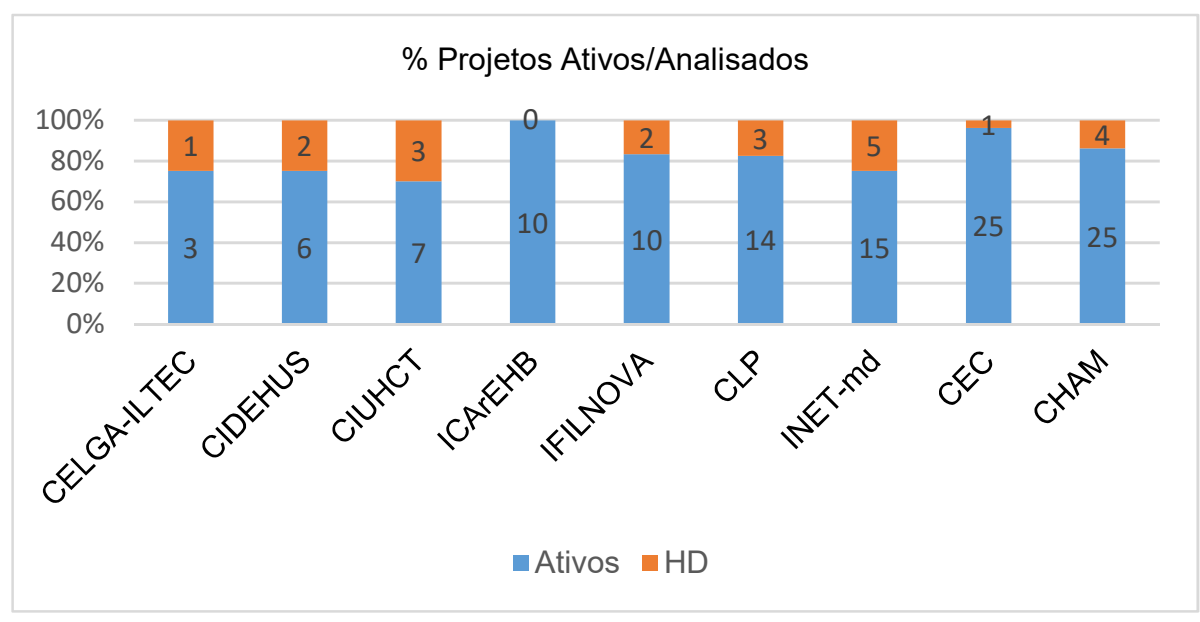

Fonte: Elaborado pelo autor.

$\mathrm{Na}$ verdade, não nos pareceu correto realizar semelhante exercício no caso das unidades em que, conforme a sua informação disponibilizada via Web, não existia uma clara demarcação daqueles que eram os seus projetos ativos (definindo um conjunto concreto) e além disso, mesmo estando identificados, por vezes não revelavam mais do que o nome do projeto ou o seu responsável.

De qualquer forma, como se pode ver, considerámos a análise de alguns projetos ativos, sobretudo dos que apresentavam informação relevante para este estudo (Tabela 1 - excerto do Anexo A). Fizemo-lo mesmo não se obtendo um número global de projetos ativos para poder realizar a análise efetuada anteriormente ou apenas os pudéssemos validar como estando ativos de forma indireta, mediante a leitura de toda a informação disponibilizada nos sítios Web.

Quadro 4 - Lista das unidades de I\&D sem um conjunto definido de projetos ativos ou sem informação suficiente

\begin{tabular}{|c|c|c|}
\hline Unidade I\&D & $\begin{array}{c}\mathbf{N}^{\circ} \text { Projectos em curso/activos } \\
\text { CECS }\end{array}$ & $\begin{array}{c}\mathbf{N}^{\circ} \text { Projetos } \\
\text { analisados }\end{array}$ \\
\hline CESEM & $\begin{array}{c}\text { 5 grupos de investigação - sem menção } \\
\text { específica a projetos ativos } \\
\text { específica a projectos activos }\end{array}$ & 1 \\
\hline CINEICC & $\begin{array}{c}3 \text { grupos de investigação - sem menção } \\
\text { específica a projetos ativos }\end{array}$ & 0 \\
\hline CIPsi & $\begin{array}{c}\text { 3 Unidades e 3 Laboratórios - sem menção } \\
\text { a projectos activos }\end{array}$ & 0 \\
\hline
\end{tabular}


Práticas digitais nas unidades de I\&D em Portugal: uma observação parcial da área das humanidades

Fonte: Elaborado pelo autor.

\begin{tabular}{|c|c|c|}
\hline CPUP & $\begin{array}{c}5 \text { grupos de investigação - sem menção } \\
\text { específica a projectos activos }\end{array}$ & 1 \\
\hline IHC & 57 & 1 \\
\hline ILC & $\begin{array}{c}1 \text { Projeto estratégico com } 3 \text { grupos de } \\
\text { investigação - sem menção específica a } \\
\text { projetos }\end{array}$ & 1 \\
\hline VICARTE & 2 grupos de investigação com 12 equipas & 0 \\
\hline WJCR & $\begin{array}{c}2 \text { linhas de investigação - sem menção a } \\
\text { projetos ativos }\end{array}$ & 0 \\
\hline
\end{tabular}

\subsection{Texto retirado dos sítios Web}

Realizámos uma cópia integral dos textos dos projetos disponibilizados em linha que denotavam evidências das práticas digitais. O total foram 2768 palavras e 16326 carateres. A análise desses textos foi feita através de uma ferramenta geradora de nuvens de palavras (aplicando um filtro que retirou as palavras irrelevantes) de forma a obter um resultado independente. A aplicação WordltOut ${ }^{3}$ revelou uma presença forte de palavras como digital, trabalho, dados, entre outras. A presença da palavra "digital" confirma dados de outro estudo que constatou que 35 em 38 projetos usavam as tecnologias digitais (GUARDADO; BORGES, 2012, p. 47). Anote-se o destaque da história, o que a confirma como a área científica mais identificada nos projetos analisados (Figura 1).

Figura 1: Nuvem de palavras gerada a partir dos textos dos projetos disponibilizados em linha

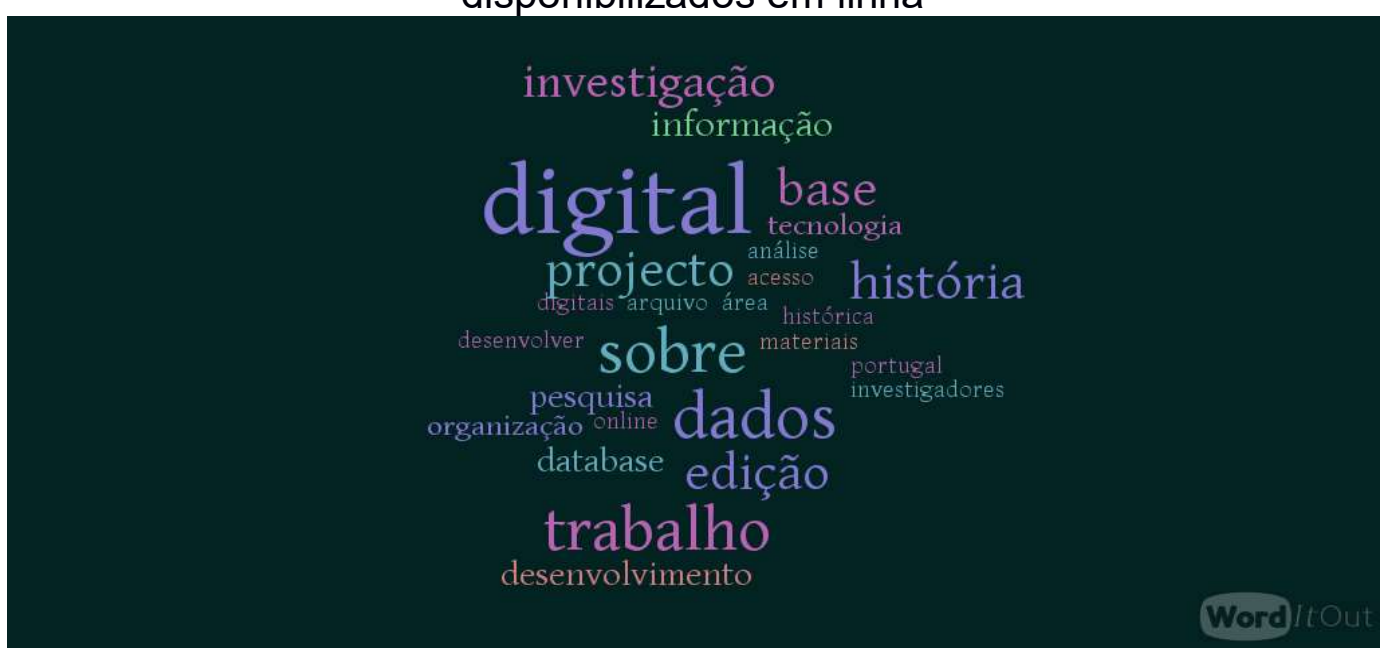

Fonte: Elaborado pelo autor.

3http://worditout.com/

Inf. Inf., Londrina, v. 22, n. 3, p. 405 - 425, set./out. 2017. http:www.uel.br/revistas/informacao/ 


\subsection{Etiquetas atribuídas (linguagem natural)}

A leitura e análise dos textos retirados dos diferentes sítios Web permitiram a atribuição de etiquetas que descrevem as práticas digitais identificadas. Esta lista (que consta integralmente no Anexo B) foi igualmente analisada com a ferramenta WordltOut produzindo uma nuvem de palavras onde se destacam dados, base, edição, digital, multimédia, sítio, entre outras (Figura 2). Na verdade, existe uma omnipresença da expressão "base de dados", o que nem sempre permite identificar o que isso significa concretamente para o projeto. Este não é um dado novo pois outro estudo identificara que $58 \%$ dos projetos usavam bases de dados (GUARDADO; BORGES, 2012, p. 47).

Figura 2 - Nuvem de palavras gerada a partir das etiquetas atribuídas

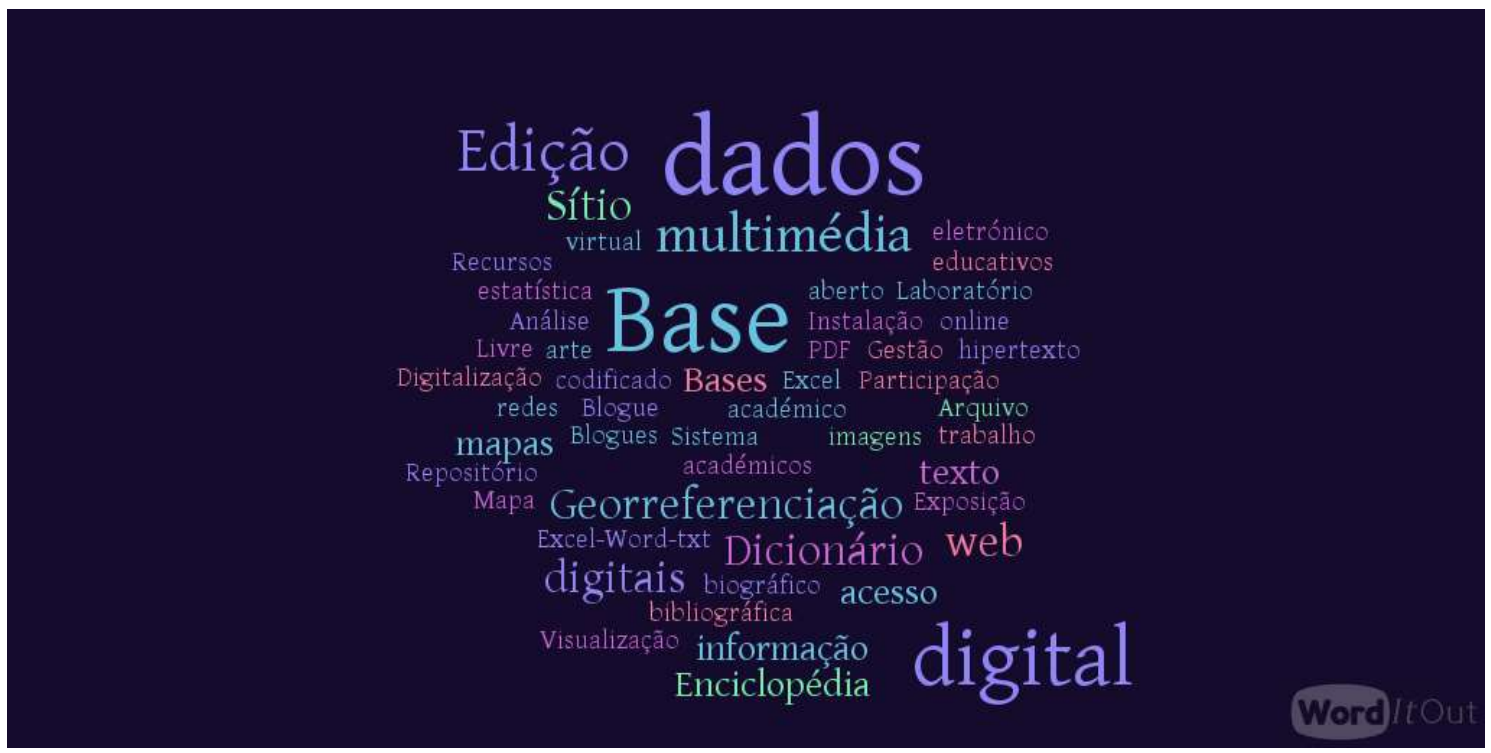

Fonte: Elaborado pelo autor. 


\subsection{Etiquetas atribuídas (linguagem natural)}

Realizámos ainda o cruzamento dos dados recolhidos com recurso à ferramenta experimental Google Fusion ${ }^{4}$, gerando alguns gráficos de redes.

Analisámos a relação entre as etiquetas atribuídas e as unidades de I\&D. A rede, cujo dinamismo e legendas não é possível apresentar na totalidade, mostrou um certo isolamento de práticas que se podem considerar de um nível básico, como a produção de um Dicionário em PDF, ou que pelo seu caráter original acabam por não serem comuns a outros nós da rede (Figura 3). Mostrou ainda a agregação de várias unidades pela partilha das etiquetas atribuídas aos seus projetos (Figura 4).

Figura 3: Relação entre as etiquetas atribuídas e as unidades de I\&D (Parcial)

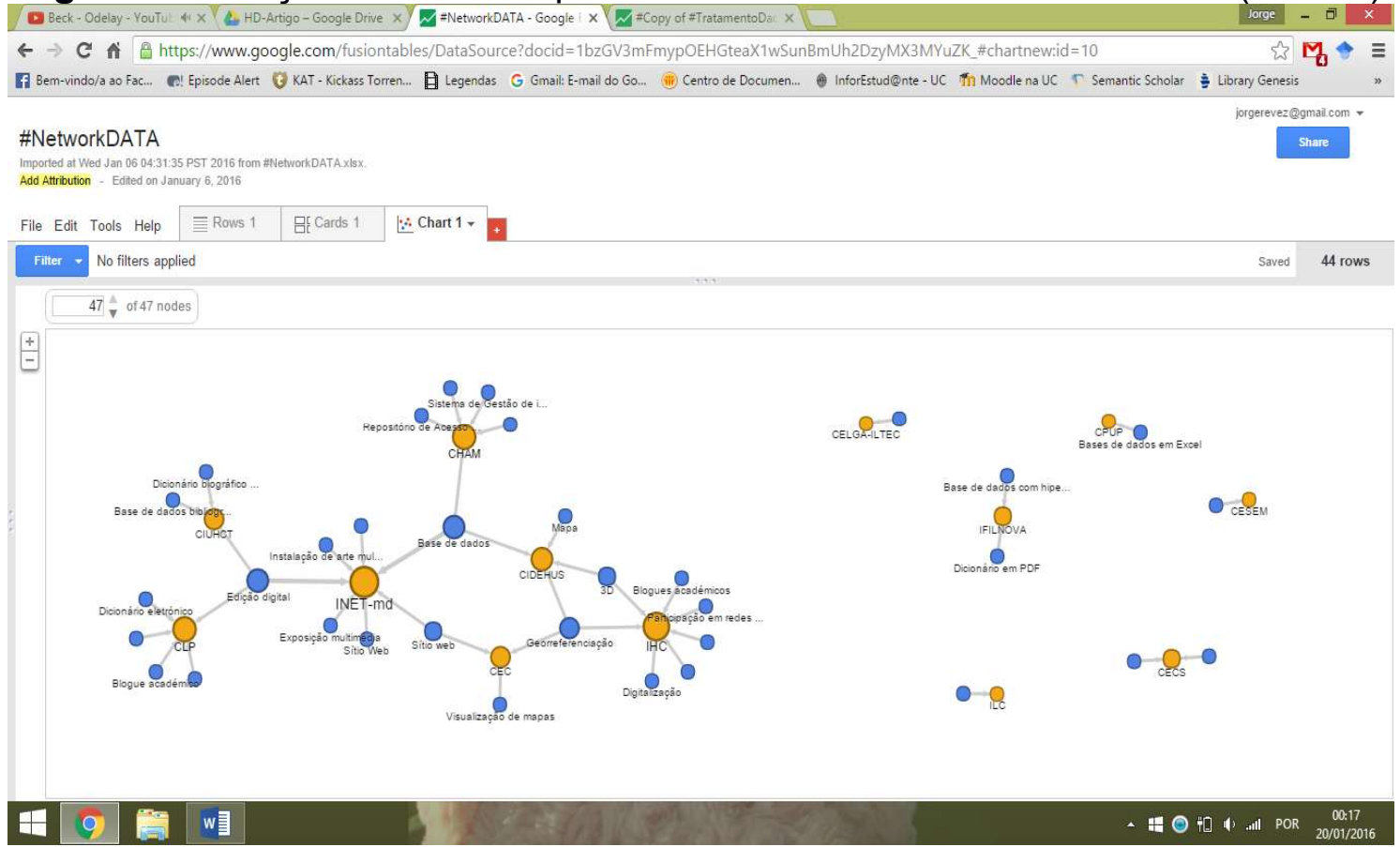

Fonte: Elaborado pelo autor.

${ }^{4}$ https://www.google.com/fusiontables/data?dsrcid=implicit 
Figura 4: Relação entre as etiquetas atribuídas e as unidades de I\&D (Parcial)

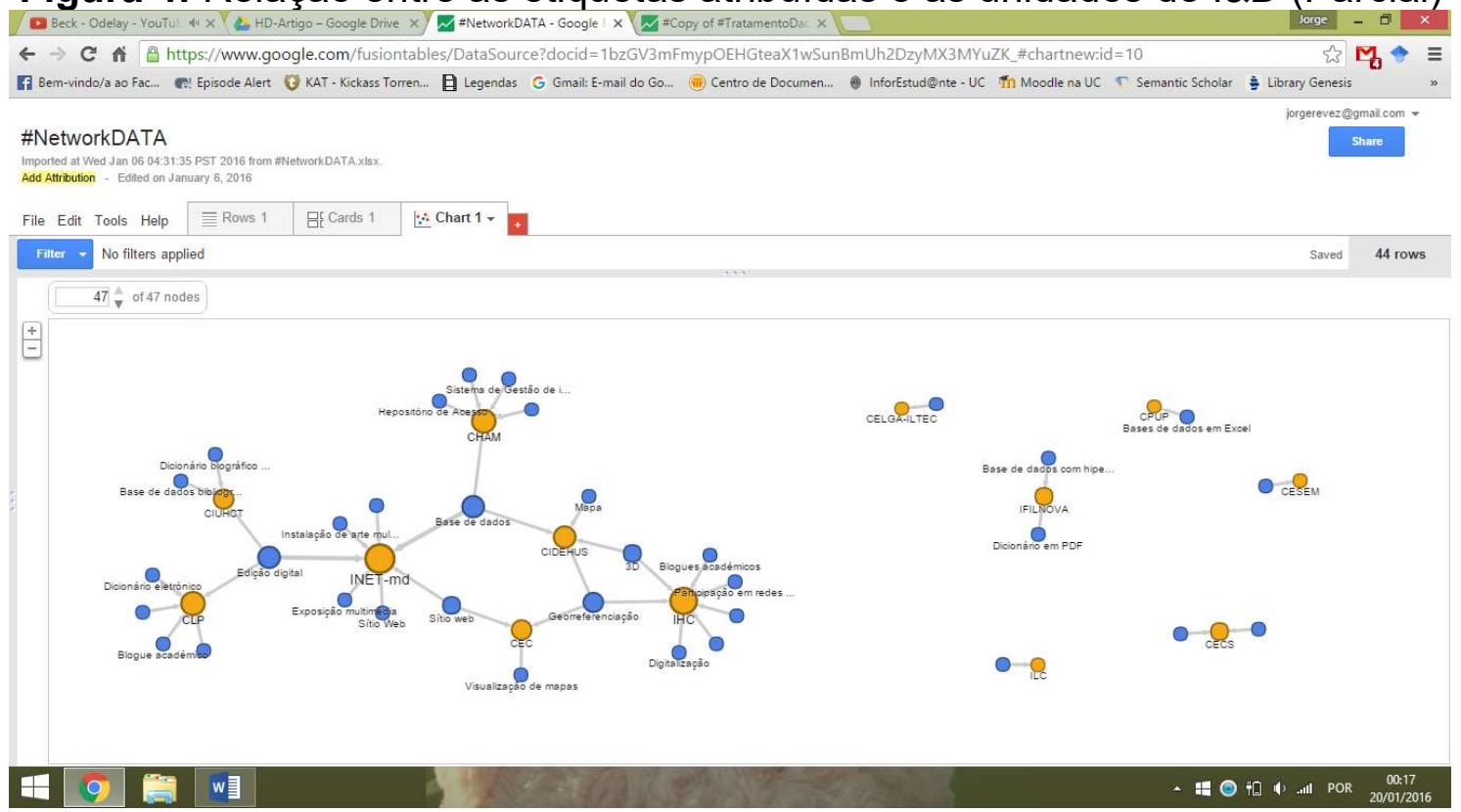

Fonte: Elaborado pelo autor.

Verificámos ainda a relação entre as etiquetas atribuídas e as áreas científicas dos projetos. A rede de etiquetas mostra uma forte relação entre as três principais áreas científicas dos projetos (História, Estudos Literários e Musicologia), criando um núcleo central. Na periferia estão as áreas com menos projetos e cujas etiquetas não estabelecem qualquer relação com o núcleo central (Figura 5). Isto pode ser explicado pela especificidade que marca os projetos ou pelo caráter básico ou menos comum de algumas das suas práticas.

Figura 5: Relação entre as etiquetas atribuídas e as áreas científicas dos

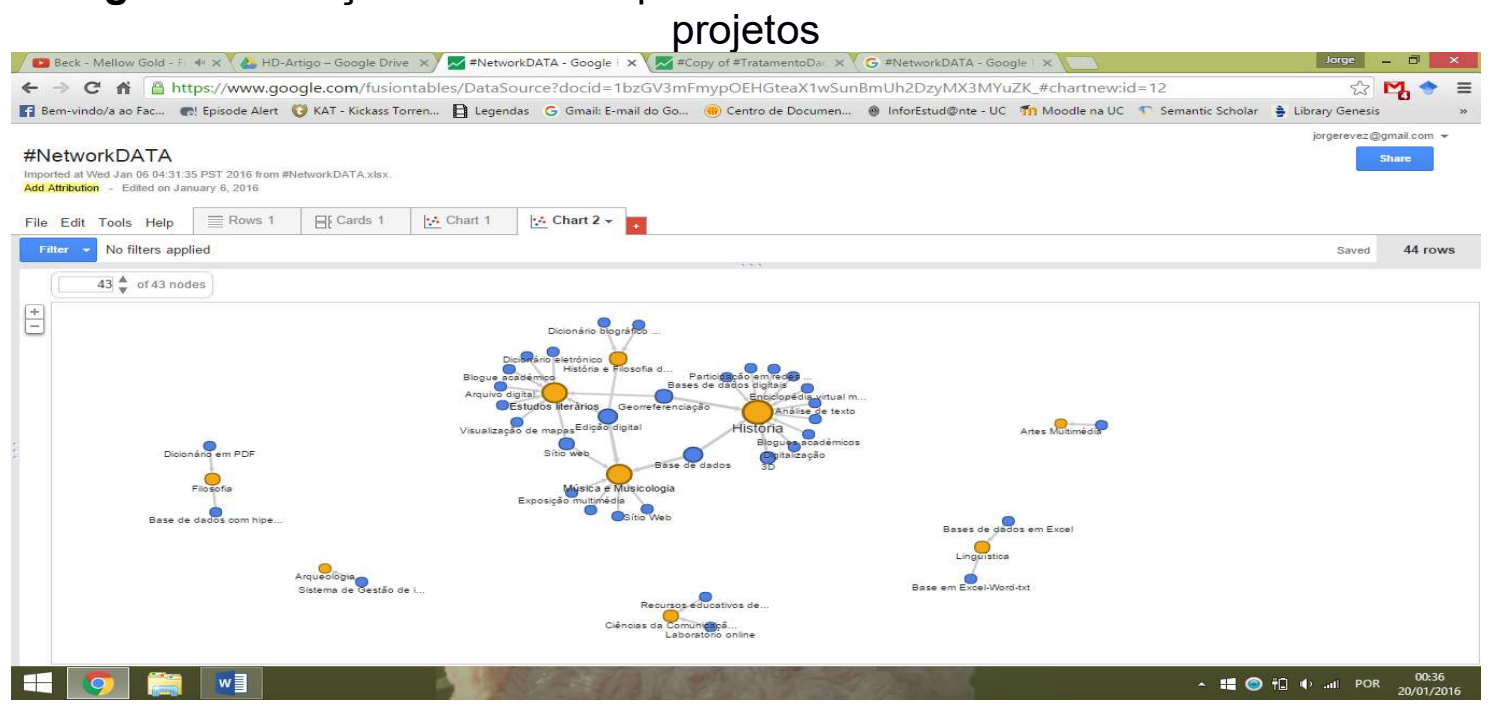

Fonte: Elaborado pelo autor. 


\section{DISCUSSÃO}

Podemos problematizar estes dados em duas perspetivas distintas: a adoção de práticas digitais nas unidades de I\&D e os motivos pelos quais este processo parece estar num estado inicial; e a especificidade da cultura epistémica (KNORR-CETINA, 1999) das Humanidades e a forma como esta se pode refletir na comunicação da ciência que está a ser feita, particularmente, na informação disponibilizada em linha.

Por um lado, em relação ao patamar digital das HD em Portugal, cremos que os dados oferecem um retrato tímido mas desigual, pois a relevância detectada em algumas unidades contrasta com uma ausência (pelo menos aparente) de práticas digitais noutras estruturas. Avançando algumas hipóteses explicativas, que teriam sempre de ser melhor estudadas e analisadas, diríamos que são quatro as variáveis possíveis:

a) O desenvolvimento recente da literacia digital dos investigadores, agravado por uma cultura digital que está centrada nas tecnologias e/ou equipamentos e não na melhoria constante de processos;

b) A lentidão da modernização das unidades de investigação em Portugal, sobretudo pela escassez de equipas multidisciplinares com especialistas de informática, gestores de ciência, entre outros;

c) A resistência dos investigadores às mudanças tecnológicas e aos efeitos de escala que as redes de informação podem proporcionar;

d) As dificuldades de financiamento para projetos de grande envergadura ou a incapacidade para angariar os meios necessários para os realizar.

Por outro lado, a cultura epistémica das Humanidades pode influenciar as formas de comunicação da ciência? É preciso ter em conta que não referimos apenas a relação com a sociedade mas também a comunicação com as outras unidades e grupos de investigação a operar nesta área. Como vimos atrás, Mullen (2010) destacou a capacidade inclusiva como uma marca do ethos das humanidades digitais.

Para diversos autores, a importância da partilha é absolutamente decisiva e irá constituir-se como uma importante mudança cultural (Sample, 2013, p. 256). Da mesma forma, Guerreiro e Borbinha consideram que "o 
principal desafio é ainda a definição de modelos genéricos para a sistematização e partilha (reuso) da informação reunida, o que faz com que de momento cada projeto seja ainda como que uma ilha" (2014, p. 73).

É preciso incrementar um sentido de pertença a uma comunidade que é cada vez mais internacional e que partilha um "núcleo de princípios e de valores" como "interdisciplinaridade; acesso aberto (open source); reconfiguração das normas de copyright e de propriedade intelectual por via da promoção de licenças alternativas (Creative Commons); trabalho colaborativo" (PEREIRA, 2015, p. 117).

Genericamente, as formas de comunicação da ciência detetadas não nos pareceram as mais adequadas pois fornecem pouca informação e são pouco transparentes quanto às atividades práticas das diferentes unidades $\mathrm{e}$ projetos. Este aspeto condicionou a nossa análise e não pode deixar de ser referido como um dos aspetos onde é urgente intervir. Algumas unidades de I\&D tornam impossível uma análise aprofundada sobre o seu trabalho científico comunicado em linha. Um guia de boas práticas que pode vir a ser seguido pelas unidades portuguesas para apoiar a construção e avaliação de projetos foi publicado pela Red de Humanidades Digitales (2013), juntamente com um auto-inquérito.

Apesar de Terras (2011) ter apresentado o número de 114 infraestruturas em 24 países, não existem ainda em Portugal estruturas que se possam considerar, na definição de Zorich (2008, p. 4-5), "centros de humanidades digitais"s. Mas é importante recolher informação e aprender com as experiências internacionais, sobretudo:

- Ultrapassar a natureza fechada (silo-like) de alguns centros que produzem conhecimento ultra-especializado, sem a capacidade de integrar esses produtos em recursos digitais de larga escala, divulgando-os e tornando-os disponíveis;

${ }^{5} \mathrm{Cf}$. a ausência de organizações portuguesas no sítio da rede centerNet (http://dhcenternet.org/), "an international network of digital humanities centres". Vide RomeroFrías e Del-Barrio-García (2014). 
- Evitar que a natureza independente de alguns centros resulte na sobreposição de atividades e de produção de conhecimento; esta redundância desperdiça os recursos financeiros escassos das Humanidades;

- Exigir um esforço colaborativo de criação de ciber-infraestruturas que beneficiem toda a comunidade, sobretudo em torno da produção digital dos centros e da sua preservação (ZORICH, 2008, p. 2).

Nos dados recolhidos, encontrámos diferentes evidências que testemunham um percurso interessante: alguns autores partem da investigação em Humanidades às práticas digitais, das práticas digitais à produção de conhecimento e à lecionação em HD. Isto é claro no caso de Daniel Alves do IHC (Seminários e Unidades Curriculares lecionados, artigos internacionais, participação em redes internacionais) e de Manuel Portela e outros autores do CLP (artigos internacionais, Summer School). No caso do IHC merece um destaque especial o acolhimento da única linha de investigação em HD identificada.

É provável que uma análise mais profunda e comparada com outros contextos de investigação, que tivesse em conta a criação de redes e em rede, como a Aracne: Red de humanidades digitales y letras hispánicas (Espanha) ${ }^{6}$, acentuasse a nossa percepção do caráter inicial em que se encontra o ofício digital em Portugal. Contudo, a questão-chave não reside no tornar tudo digital, criando bibliotecas digitais com informação inútil, mas na criação de uma infraestrutura académica digital que transforme as Humanidades como área do saber, sob a liderança dos académicos que fizeram e hão de fazer a investigação em Humanidades (BORGMAN, 2009, pr. 67). No mesmo sentido, Pereira reconhece que "as mudanças em curso na Academia são, regra geral, muito mais de ordem cultural do que propriamente tecnológica" (2015, p. 122). Mesmo toda a vertigem de mudança que estamos a experimentar terá de ser matizada: "Fórmulas como sociedade digital, cultura digital ou Humanidades Digitais tenderão a perder sentido com o tempo, pois mais não são do que

${ }^{6}$ Cf. http://www.red-aracne.es/presentacion 
constructos produzidos num quadro de profunda transformação epistémica" (2015, p. 133-134).

\section{CONCLUSÃO}

Procurámos neste exercício contribuir para a caracterização de uma realidade que ainda é um campo por explorar. Como repararam Guardado e Borges, "the use of new technologies by the scientific community is still quite unknown in Portugal" (2012, p. 49). Conhecer as práticas digitais das unidades de I\&D permite começar a compreender não apenas o uso presente mas a forma como estas organizações estão a transformar-se. Ainda que a adoção pareça estar num estado inicial, existem claras evidências de progresso. A observação da informação disponibilizada na Web pelas unidades de investigação revelou-se uma tarefa difícil mas estimulante. Os resultados da análise a 25 projetos ativos e a uma linha de investigação mostram que as práticas digitais estão disseminadas e comunicadas de forma incipiente e que a transformação preconizada pelo campo das HD ainda se encontra numa fase inicial em Portugal.

Na nossa análise sobressai o problema da comunicação das unidades de I\&D com o seu campo científico, elemento fundamental para a geração de sinergias, partilha de esforços e aumento da visibilidade. Parece-nos que a criação e o desenvolvimento de uma base de informação pública, eventualmente gerida pela FCT, permitiria centralizar, organizar e estruturar um conjunto de informações que está inutilmente disperso. A possibilidade de recolher informação sistematizada sobre 0 trabalho que está a ser desenvolvido seria uma ferramenta inestimável. Estamos em crer que a informação habitualmente enviada para as agências financiadoras pode assim ter outro tipo de tratamento e consequente impacto.

Parece-nos que não é demais insistir também na urgência de melhorar as formas de comunicação com o público. Só uma comunicação eficaz poderá revelar as vantagens do investimento que a sociedade está a fazer na investigação científica das Humanidades. Isto é importante na medida em que pode demonstrar a capacidade das Humanidades sobreviverem num ambiente 
altamente competitivo, em que é muitas vezes questionado o seu papel social e o seu contributo para o desenvolvimento económico.

\section{REFERÊNCIAS}

ALVES, D. From «Humanities and Computing» to «Digital Humanities»: Digital Humanities in Portugal with a focus on Historical Research. H-Soz-Kult, 24-102014. Disponível em: <http://www.hsozkult.de/debate/id/diskussionen-2455>. Acesso em: 20 dezembro 2015.

BERRY, D. M. (Ed.). Understanding digital humanities. Houndmills, Basingstoke, Hampshire: Palgrave Macmillan, 2012.

BORGMAN, C. L. The Digital Future is Now: a Call to Action for the Humanities. DHQ: Digital Humanities Quarterly, v.3, n.4, 2009.

BULGER, M. et al. Reinventing research? information practices in the humanities: a Research Information Network report. London: RIN, 2011.

GUARDADO, M. C.; Borges, M. M. Some trends in electronic publication and open access in Portuguese history journals. Information Services \& Use, v.31, p.235-241, 2011.

GUARDADO, M. C.; BORGES, M. M. Digital History in Portugal: a survey. In: Tokar, A. et al. (Ed.). Science and the Internet. Düsseldorf: Düsseldorf University Press, 2012. p.43-54.

GUERREIRO, D.; BORBINHA, J. L. Humanidades Digitais: novos desafios e oportunidades. Cadernos BAD, n.1, p.63-78, 2014.

HAYLES, N. K. How we think: transforming power and digital technologies. In: Berry, D. (Ed.). Understanding digital humanities. Houndmills, Basingstoke, Hampshire: Palgrave Macmillan, 2012.

KNORR CETINA, K. Epistemic cultures: how the sciences make knowledge. Cambridge: Harvard Univesity Press, 1999.

MULLEN, L. Digital humanities is a spectrum; or, we're all digital humanists now. 2010. Disponível em: <http://lincolnmullen.com/blog/digitalhumanities-is-a-spectrum/>. Acesso em: 20 dezembro 2015.

PARRY, D. The Digital Humanities or a Digital Humanism. In: Gold, M. K. (Ed.). Debates in the digital humanities. Minneapolis: University of Minnesota Press, 2012. p. 429-437. 
PEREIRA, P. S. Academia, Geopolítica das Humanidades Digitais e Pensamento Crítico. Matlit: Revista do Programa de Doutoramento em Materialidades da Literatura, v.3 n.1, p.111-140, 2015.

PRIANI SAISÓ, E. et al. Las humanidades digitales en español y português: un estudio de caso: DíaHD/DiaHD. Anuario Americanista Europeo, n.12, p.5-18, 2014.

RED DE HUMANIDADES DIGITALES. Guía de buenas prácticas para la elaboración y evaluación de proyectos digitales en las Humanidades. 2013. Disponível em: <http://humanidadesdigitales.net/evaluacion/>. Acesso em: 20 dezembro 2015.

ROMERO-FRÍAS, E.; DEL-BARRIO-GARCÍA, S. Una visión de las humanidades digitales a través de sus centros. El profesional de la información, v.23, n.5, p.485-492, 2014.

SAMPLE, M. The Digital Humanities is not about Building, it's about Sharing" [orig. 2011]. In TERRAS, M.; NYHAN, J.; VANHOUTTE, E. (Eds.). Defining digital humanities: a reader. Farnham: Ashgate, 2013. p. 255-257.

SVENSSON, P. The Landscape of Digital Humanities. DHQ: Digital Humanities Quarterly, v.4, n.1, 2010.

TERRAS, M. Quantifying digital humanities. London: UCL Centre for Digital Humanities, 2011.

VARNER, S.; HSWE, P. Special Report: Digital Humanities in Libraries. American Libraries, January-February, p.36-41, 2016.

ZORICH, D. A survey of digital humanities centers in the United States. Washington, D.C.: Council on Library and Information Resources, 2008.

\title{
Title
}

Digital practices of R\&D units in Portugal: a partial observation in the humanities

\begin{abstract}
Introduction - On which digital level are Portuguese Humanities? The answer to this question requires an approach to the Digital Humanities theoretical framework and an application of this framework to the situation of the Portuguese scientific research. It is the first crossover study (not restricted to a discipline) on digital practices in the Humanities in Portugal.

Purpose - The goal is to analyze the digital practices in the Humanities in Portugal. Methodology - A sample of 18 Portuguese R\&D units working in the Humanities was observed and their ongoing research projects were identified. The most important projects for an inventory construction were considered with an evidence categorization regarding digital tools embedded in scientific work and research outputs.
\end{abstract}


Results - The analysis of 25 active projects and one research line shows that digital practices are poorly disseminated and communicated.

Conclusions - The transformation encouraged by Digital Humanities is still at an early stage in Portugal.

Keywords: Digital Humanities. Scientific Research. Humanities. R\&D units. Portugal.

\section{Titulo}

Prácticas digitales en las unidades de I+D en Portugal: una observación parcial de las humanidades

\section{Resumen}

Introducción - ¿A qué nivel digital están las Humanidades portuguesas? La respuesta a esta pregunta requiere un enfoque de marco teórico de las Humanidades digitales y una aplicación de este marco a la situación de la investigación científica portuguesa. Es el primer estudio cruzado (no restringido a una disciplina) sobre las prácticas digitales en las humanidades en Portugal.

Objetivo - El objetivo es analizar las prácticas digitales en las Humanidades en Portugal.

Metodología - Nos fijamos en una muestra de 18 unidades de I+D portuguesas que trabajan en el campo de las Humanidades e identificamos sus proyectos de investigación en curso. Fueron considerados los más importantes proyectos para la construcción de un inventario y clasificación de las pruebas de la incorporación de las herramientas digitales en el trabajo científico y la creación de sus productos finales.

Resultados - Los resultados del análisis de 25 proyectos activos y una línea de investigación muestran que las prácticas digitales son difundidas y comunicadas de modo rudimentario.

Conclusiones - La transformación defendida por el campo de las Humanidades Digitales se encuentra todavía en una etapa temprana en Portugal.

Palabras clave: Humanidades digitales. Investigación científica. Humanidades. Unidades de I+D. Portugal.

Recebido em: 18.04.2016

Aceito em: 13.12.2017 